\title{
Schematic representations for teaching the procedure of optical amplification in fiber amplifiers
}

\section{Cüneyt Berkdemir, Sedat Özsoy}

Cüneyt Berkdemir, Sedat Özsoy, "Schematic representations for teaching the procedure of optical amplification in fiber amplifiers," Proc. SPIE 9664, Ninth International Topical Meeting on Education and Training in Optics and Photonics, 966417 (24 October 2005); doi: 10.1117/12.2207767

Event: Ninth International Topical Meeting on Education and Training in Optics and Photonics, 2005, Marseille, France 


\title{
Ref ETOP083
}

\section{Schematic representations for teaching the procedure of optical amplification in fiber amplifiers}

\author{
Cüneyt Berkdemir (OSA Member) and Sedat Özsoy
}

Department of Physics, Faculty of Arts and Sciences, Erciyes University, Kayseri, Turkey, 38039, berkdemir@erciyes.edu.tr and ozsoys@erciyes.edu.tr

\begin{abstract}
We present the schematic representations for teaching the procedure of optical amplification in erbium and praseodymium doped fiber amplifiers by using rate equations models. The behaviour of these types of amplifiers is the same as three (for erbium ion) and four (for praseodymium ion) levels atomic systems, respectively. Most of the important characteristics of these amplifiers can be obtained from these simple models and their underlying assumptions. Hence, we can make use of these approaches for teaching the purposes of procedures in fiber amplifiers. For this aim, the gain performances of $\mathrm{Er}^{3+}$ and $\mathrm{Pr}^{3+}$-doped fiber amplifiers are investigated at the educational level, using the temperature-dependent rate equation model.
\end{abstract}

\section{1- Introduction}

\section{Summary}

Fiber optic technology has been advancing at a surprising pace during the last three decades. It was about fifteen years ago that researchers showed the great potential of optical fiber doped with the rare-earth elements such as erbium and praseodymium, for application in long haul communications. Optical amplifiers have been successfully incorporated in the field of fiber communication, accelerating the rate at which optical links are being deployed over the continents and the transoceanics. Today, this technology is fast becoming a major part of optical communications infrastructure in many countries around the world [1, 2]. Therefore, in order to learn this technology at university level, we need to understand the procedure of optical amplification in fiber amplifiers from basic to advance. From this point of view, the education and training programs will make easy to understand the procedure of optical amplification in fiber amplifiers.

\section{2- Schematic Representations of Amplification}

This work will enable us to understand the basic physical concepts underlying three and four level fiber amplifiers and obtaining the signal gain of amplifiers by using rate equation models. Figure 1 shows the schematic representations of the energy levels and amplifications mechanism for Er3+ (left hand side of picture) and Pr3+ (right hand side of picture) ions doped in glass hosts. 

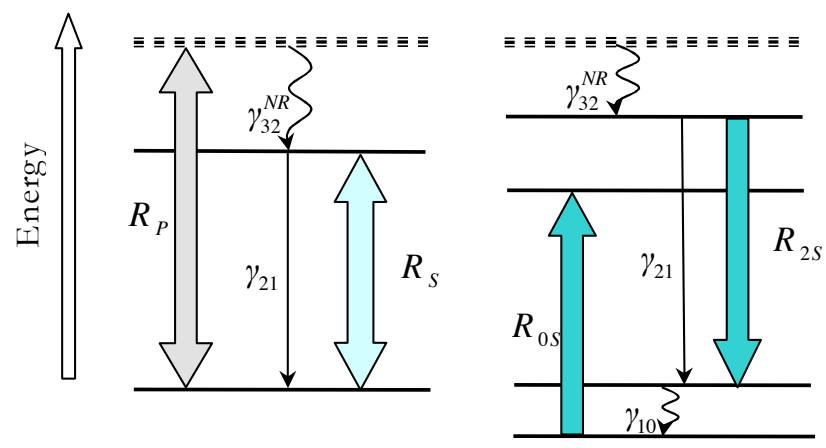

Level 3

$E_{3}, N_{3}$

Level 2

$E_{22}, N_{22}$

$E_{21}, N_{21}$

Level $1 \quad E_{1}, N_{1}$

Level $0 \quad E_{0}, N_{0}$

Figure 1: Energy levels and amplification mechanism for Er3+ (left hand side of picture) and Pr3+ (right hand side of picture)-ions in glass hosts.

The energies and populations of three and four level amplifiers can be described by $E_{0}, E_{1}, E_{2}\left(=E_{21}+E_{22}\right), E_{3}$ and $N_{0}, N_{1}, N_{2}\left(=N_{21}+N_{22}\right), N_{3}$, respectively, for the thermal equilibrium. By definition, levels 0 and 1 are the lowest or ground state, level 2 is the metastable state, and level 3 is the pump level. The stimulated transition probability $R_{p}$ is the probability of exciting the ion from level 1 to level 3 for erbium, and from level 0 to level 3 for praseodymium by pumping, and $R_{s}$ will be referred to the stimulated transition probability between $E_{2}$ and $E_{1}$.

$$
\begin{array}{l|l}
\frac{d N_{3}}{d t}=R_{p}\left(N_{1}-N_{3}\right)-\gamma_{32}^{N R} N_{3}, & \begin{array}{l}
\frac{d N_{3}}{d t}=R_{p} N_{0}-R_{p} N_{3}-\gamma_{32}^{N R} N_{3}, \\
\frac{d N_{2}}{d t}=-R_{s}\left(N_{2}-N_{1}\right)-\gamma_{21} N_{2}+\gamma_{32}^{N R} N_{3},
\end{array} \\
\frac{d N_{1}}{d t}=-R_{p}\left(N_{1}-N_{3}\right)+\gamma_{21} N_{2}+R_{s}\left(N_{2}-N_{1}\right) & \begin{array}{l}
\frac{d N_{21}}{d t}=R_{0 S} N_{0}-R_{2 S} N_{22}-N_{22} \gamma_{21}+\gamma_{32}^{N R} N_{3} \\
\frac{d N_{1}}{d t}=R_{2 S} N_{22}+\gamma_{21} N_{22}-\gamma_{10} N_{1} \\
\frac{d N_{0}}{d t}=R_{p} N_{3}-R_{p} N_{0}-R_{0 S} N_{0}+\gamma_{10} N_{1} .
\end{array},
\end{array}
$$

Let us now consider the steady-state situation, where the populations are time invariant, i.e., $d N_{i} / d t=0 \quad(i=0,1,2,3)$;

$$
N_{2}=N \frac{R_{p}+R_{s}}{R_{p}(1+2 \beta)+R_{s}(2+\beta)+\gamma_{21}} \quad N_{1}=N \frac{R_{p} \beta+R_{s}+\gamma_{21}}{R_{p}(1+2 \beta)+R_{s}(2+\beta)+\gamma_{21}} ; \quad \text { for Er3+- }
$$

doped fiber,

$$
N_{22}=N \frac{R_{p}}{\gamma_{21}+R_{p}(1+1 / \beta)+R_{2 S}} \quad N_{0}=N \frac{\gamma_{21}+S_{2 S}}{\gamma_{21}+R_{p}(1+1 / \beta)+S_{2 S}} ; \quad \text { for Pr3+- }
$$

doped fiber. 
Here, $\beta$ quantity is involved in the issue because of the $E_{2}$ and $E_{3}$ levels of $\mathrm{Er}^{3+}$ and the $E_{22}$ and $E_{21}$ levels of $\operatorname{Pr}^{3+}$-doped fibers are very closely spaced according to the Boltzmann's population relation $N_{m} / N_{m-1}=e^{-\Delta E_{m} / k_{B} T}$ $\left(\Delta E_{m}=E_{m}-E_{m-1}, k_{B}=1,38 \times 10^{-23} \mathrm{~J} / \mathrm{K}\right)$. We consider an absorbing medium for the description of the signal gain. The signal gain is given by $\mathrm{G}=\exp \left[\Gamma_{\mathrm{S}}\left(\sigma_{21} \mathrm{~N}_{2}-\sigma_{12} \mathrm{~N}_{1}\right) \mathrm{L}\right]$ for Er3+-doped fiber [3] and by $\mathrm{G}=\exp \left[\Gamma_{\mathrm{s}} \sigma_{2 \mathrm{~S}} \mathrm{~N}_{22} \mathrm{~L}\right]$ for Pr3+-doped fiber amplifiers [1]. Here, L denotes the fiber amplifier length and the other parameters represent the standard fiber parameters.

\section{3- Conclusions}

We have introduced simple schematic representations for energy levels and amplification mechanisms, and used the basic rate equation models, including the temperature effect to obtain the signal gain of the erbium and praseodymium-doped fiber amplifiers at university level. In addition, we have shown the possibility of deriving an analytical solution of the rate equations in some practical temperature ranges to understand the gain performance of both fiber amplifiers.

\section{Acknowledgment}

This study is supported by Scientific Research Projects Council (SRPC) of Erciyes University under Grant No FBA-04-01.

\section{References}

1- P.C. Becker, N. Olsson, J. Simpson, Erbium-Doped Fiber Amplifiers: Fundamentals and Technology, Academic Press, San Diego, (Copyright by Lucent Technologies) 1999.

2- K. Amano and Y. Iwamoto, Optical Fiber Submarine Cable System, Journal of Lightwave Technology., vol. 8, no. 4, 595, (1990).

3- C. Berkdemir and S. Özsoy, The temperature dependent performance analysis of EDFAs pumped at $1480 \mathrm{~nm}$ : A more accurate propagation equation, Optics Express, 13 (13), 5179, (2005). 